\title{
HI STUDIES OF THE SCULPTOR GROUP GALAXIES
}

\author{
Claude Carignan, Université de Montréal \\ and \\ Daniel Puche, Very Large Array, NRAO
}

\begin{abstract}
Results from large-scale mapping of the HI gas in the Sculptor group are presented. From our kinematic analysis, a mean global $\left(M / L_{B}\right) \simeq 9 \mathrm{M}_{\odot} / \mathrm{L}_{\odot}$ (at the last observed velocity point) is found for the individual galaxies. This is only a factor $\sim 10$ smaller than the $\left(M / L_{B}\right)_{d y n} \simeq 90 \mathrm{M}_{\odot} / \mathrm{L}_{\odot}$ derived from a dynamical study of the whole group. The parameters derived from the mass models suggest that most of the unseen matter has to be concentrated around the luminous galaxies. Under the assumption that the Sculptor group is a virialized system and that all the mass is associated with the galaxies, an upper limit of $\sim 40 \mathrm{kpc}$ is derived for the size of the dark halos present in the five late-type spirals of the group.
\end{abstract}

\section{Introduction}

Dynamical studies of individual galaxies, groups of galaxies, and clusters of galaxies have led to a large variety of sometimes contradictory values of dynamical mass-to-light ratios $\left(M / L_{B}\right)$. The present situation can be summarized as follows. According to the scale considered, determinations of $M / L_{B}$ range from 1 to $500 \mathrm{M}_{\odot} / \mathrm{L}_{\odot}$. In this interval, the most cited numbers are $\simeq 2-10$ for individual galaxies (from rotation curves of spirals), $\simeq 30-100$ for binaries and small groups, and $\simeq 200$ for rich clusters (Faber and Gallagher 1979). The obvious conclusion is that there is a large quantity of dark matter (DM) present in these systems. However, the question of whether this unseen matter is associated with the individual galaxies, is more uniformly distributed, or both, still remains to be settled.

The closest group of galaxies outside the Local Group for which we can study the kinematics and the distribution of DM is the Sculptor group. It covers an area of $20^{\circ}$ in diameter centered at $\alpha=0^{h} 30^{m}$ and $\delta=-30^{\circ}$. Prior to this study, the kinematical data on the Sculptor group galaxies were of unequal quality, sensitivity and resolution (optical Fabry-Pérot, single dish HI, Owens Valley interferometry ...). We now present a homogeneous data set from new HI line observations obtained at the Very Large Array (VLA). Because Sculptor is a nearby group $(\sim 2.5 \mathrm{Mpc})$, the resolution of $30^{\prime \prime}$ to $60^{\prime \prime}(\sim$ $500 \mathrm{pc}$ ), obtained with the C/D configuration, is ideal to allow detailed modeling of the mass distribution. 


\section{Dynamical Study of the Sculptor Group}

The first step of the analysis is to determine the membership of the group. For this, we use the dynamical mass estimators derived by Heisler, Tremaine, and Bahcall (1985) for a system of self-gravitating objects of comparable masses. This approach is justified since all the galaxies have equivalent absolute magnitudes $\left(\left\langle M_{B}\right\rangle \simeq-18.2 \pm 0.6\right)$. This shows the group to be mainly composed of five members: NGC 7793, NGC 55, NGC 247, NGC 253, and NGC 300 (Puche and Carignan 1988). Two other galaxies, NGC 45 and NGC 24 , which are observed in the general direction of the Sculptor group are found to be more distant objects. As for the dwarfs known to belong to Sculptor (e.g. SDig: Lausten et al. 1977), they need not be considered because of their small contribution to the dynamics of the group.

Assuming that the Sculptor group is a virialized system, a total dynamical mass of $2.0 \times 10^{12} \mathrm{M}_{\odot}$ is derived. This is an upper limit since the virialized state of the group can be questioned (de Vaucouleurs 1959). This gives a total $\left(M / L_{B}\right)_{d y n} \simeq 90 \mathrm{M}_{\odot} / \mathrm{L}_{\odot}$.

\section{Kinematic Studies of the Sculptor members}

All the galaxies were observed in $\mathrm{HI}$ at the VLA. NGC 7793, being on the far side of the group could be studied with only one field (HPBW $\simeq 32^{\prime}$ ). However, for NGC 55 and NGC 300, which are on the near side of the group, a 'mosaicing' technique had to be used to get sufficient sensitivity in the outer parts. For NGC 55, which is nearly edgeon, two fields were sufficient while five fields had to be combined for NGC 300 . The two other galaxies, NGC 247 and NGC 253, which are at the mean distance of the group, were observed with only one field. Since the detected HI extends to $\sim 30^{\prime}$, they clearly need to be reobserved in two fields.

The velocity fields of the five galaxies were analysed using a full tilted ring model. Important warps of the HI plane are clearly seen for NGC 7793, NGC 253 and NGC 300. Using the derived rotation curves, the mass distribution was analysed for each galaxy using multi-component models (Carignan and Freeman 1985). The contribution from the stellar disk is calculated by a straight inversion of the luminosity profile (Kalnajs 1983) where the $\left(M / L_{B}\right)_{*}$, assumed constant throughout the disk, is the only free parameter. The surface density profile of the HI gas is obtained by elliptically averaging the total HI maps ( $\times 4 / 3$ to account for primordial $\mathrm{He}$ ). The DM halo is modeled by an isothermal sphere potential (Carignan 1985). This component has two free parameters: a radial scaling $r_{c}$ (core radius) and a velocity scaling $\sigma$ (one dimensional velocity dispersion). The central density is then given by $\rho_{0}=9 \sigma^{2} / 4 \pi G r_{c}^{2}$.

Using the results of the mass models, a mean $\left(M / L_{B}\right)_{\text {global }}=9.2 \pm 4.6$ is found for the five members of Sculptor at the last measured velocity point of the rotation curve. The $\left(M / L_{B}\right)_{\text {global }}$ range from 5 for NGC 7793 to 15 for NGC 253. It is important to notice that the mean $\left(M / L_{B}\right)_{g l o b a l}$ is only a factor 10 smaller than the $\left(M / L_{B}\right)_{d y n}$ obtained for the group as a whole. 


\section{Discussion}

Combining the results from the dynamical analysis of the Sculptor group with the results obtained from the kinematics of its individual members, two main conclusions can be reached. The mass models give us a measure of the mean density of DM in the five galaxies. At the last measured velocity point of the rotation curves, we find $\left\langle\rho_{D M}\right\rangle \simeq$ $5.0 \times 10^{-3} \mathrm{M}_{\odot} \mathrm{pc}^{-3}$. If the $\mathrm{DM}$ was uniformly distributed in the group, its mean density would only be $\simeq 5.0 \times 10^{-6} \mathrm{M}_{\odot} \mathrm{pc}^{-3}$. This gives a density enhancement for the DM in the halos of $\sim 10^{3}$ which suggests that a large fraction of the unseen matter has to be concentrated in the neighborhood of the luminous galaxies.

Following this result, if one assumes that all the DM is associated with the optical galaxies and that the Sculptor group is a virialized system, the dark halos only need to extend to $\sim 40 \mathrm{kpc}$ so that each galaxy has a $\left(M / L_{B}\right)_{g l o b a l} \simeq 90$, which is the $\left(M / L_{B}\right)_{d y n}$ derived for the whole group. Because Sculptor may not be virialized, this value of $40 \mathrm{kpc}$ is an upper limit.

Finally, the results from this study cannot easily be used to make inferences on the possible nature of DM. The uncertainties on the modeling of the mass distribution are still too large to derive severe constraints (see e.g. Lake 1989). Suffice to say that the mean density of DM in the halos of the Sculptor galaxies $\rho_{D M} \simeq 0.005 \mathrm{M}_{\odot} \mathrm{pc}^{-3}$ corresponds to only $\simeq 5 \mathrm{pc}^{-3}$ Jupiter size bodies. This is very low considering, for example, that brown dwarfs, which are serious baryonic DM contenders, could have masses an order of magnitude greater than Jupiter; hence, the density of such objets would only need to be $\leq 1 \mathrm{pc}^{-3}$.

\section{References}

Carignan, C. 1985. Ap. J., 299, 59.

Carignan, C., and Freeman, K.C. 1985. Ap. J., 294, 494.

Faber, S.M., and Gallagher, J.S. 1979. Ann. Rev. Astron. Astrophys., 17, 135.

Heisler, J., Tremaine, S., and Bahcall, J.N. 1985. Ap. J., 298, 8.

Kalnajs, A. 1983. In IAU Symp. 100, Internal Kinematics and Dynamics of Galaxies, ed. E. Athanassoula (Dordrecht: Reidel), p. 87.

Lake, G. 1989. A. J., 98, 1253.

Laustsen, S., Richter, N., van der Lans, J., West, R.M., and Wilson, R .N. 1977. Astron. Astrophys., 54, 639.

Puche, D., and Carignan, C. 1988. A. J., 95, 1025. 